\title{
A Lisfranc Fracture-Dislocation in a Football Player
}

\author{
Marc I. Harwood, MD, and Steven M. Raikin, MD
}

Injuries to the tarsometatarsal joint, also known as Lisfranc injuries, are uncommon and can be difficult to diagnose. These injuries have a high potential for causing substantial disability related to posttraumatic osteoarthritis. As a result, it is important to be alert for these injuries when evaluating patients after acute foot trauma. This case report describes a collegiate athlete with a Lisfranc fracture-dislocation. Relevant aspects of the physical examination, diagnostic imaging, and treatment considerations are discussed.

\section{Case Report}

The patient, a 22 year-old male collegiate football player, was examined on the field after sustaining an injury to his right foot. After falling on a plantarflexed, fixed foot, an axial load was applied to the posterior aspect of the patient's right heel. He developed immediate pain and swelling over the dorsum of the foot, and he was unable to bear weight on his foot. His medical and surgical history were unremarkable.

When the patient was examined, he was in extreme discomfort. He had considerable swelling over the dorsum of the midfoot. His foot was diffusely tender with areas of maximal tenderness over the first and second tarsometatarsal joints, the medial cuneiform, and between the first and second metatarsal heads. The foot was otherwise neurologically intact.

The patient was sent to the local emergency department for radiographs immediately after the injury. Non-weight-bearing anteroposterior, lateral, and oblique radiographs were obtained. The physicians caring for the patient in the emergency

Submitted, revised, 10 September 2002.

From the Department of Family Medicine (MIH), the Department of Orthopaedic Surgery (SMR), and the Orthopaedic Foot \& Ankle Service (SMR), Jefferson Medical College, Thomas Jefferson University Hospital, Philadelphia. Address reprint requests to Marc I. Harwood, MD, Department of Family Medicine, Jefferson Medical College, Thomas Jefferson University Hospital, 1015 Walnut St., Suite 401, Philadelphia, PA 19107. department, including the on-call radiologist, did not observe any abnormalities on the initial films. The patient was released from the emergency department with crutches and a postoperative shoe. He was told that he had a severe sprain of his foot. When the patient brought the radiographs to the training room the next day, a fracture of the medial cuneiform was seen in addition to $2 \mathrm{~mm}$ of widening between the medial and middle cuneiform articulation, findings consistent with a Lisfranc fracture-dislocation (Figure 1).

The patient's foot was placed in a fracture boot, and he was assigned to strict non-weight-bearing status. He was then referred to a foot and ankle surgeon, who took him to the operating room 4 days after the injury for open reduction and internal fixation of both the medial cuneiform fracture and the Lisfranc dislocation. A cannulated screw was placed from the medial aspect of the medial cuneiform across the Lisfranc articulation into the base of the second metatarsal. A second screw was placed across the medial cuneiform into the middle cuneiform (Figure 2). The patient was instructed to remain non-weight bearing, and his foot was put in a cast to immobilize it. He was then given a fracture boot to wear for 6 weeks. He was gradually weaned from his fracture boot without difficulty. The hardware was removed 19 weeks after surgery. At 42 weeks after surgery and 22 weeks after removal of the screws, the patient was asymptomatic and was given permission to participate in all activities without restrictions. He participated in preseason conditioning without incident and was planning to compete in the regular season.

\section{Discussion}

The Lisfranc joint complex is composed of the articulations between the tarsal and metatarsal bones. Transverse ligaments join the bases of all the metatarsals with the exception of the articulation between the first and second metatarsals. ${ }^{1,2}$ The Lisfranc ligament spans the medial cuneiform and the base of the second metatarsal. The base of 

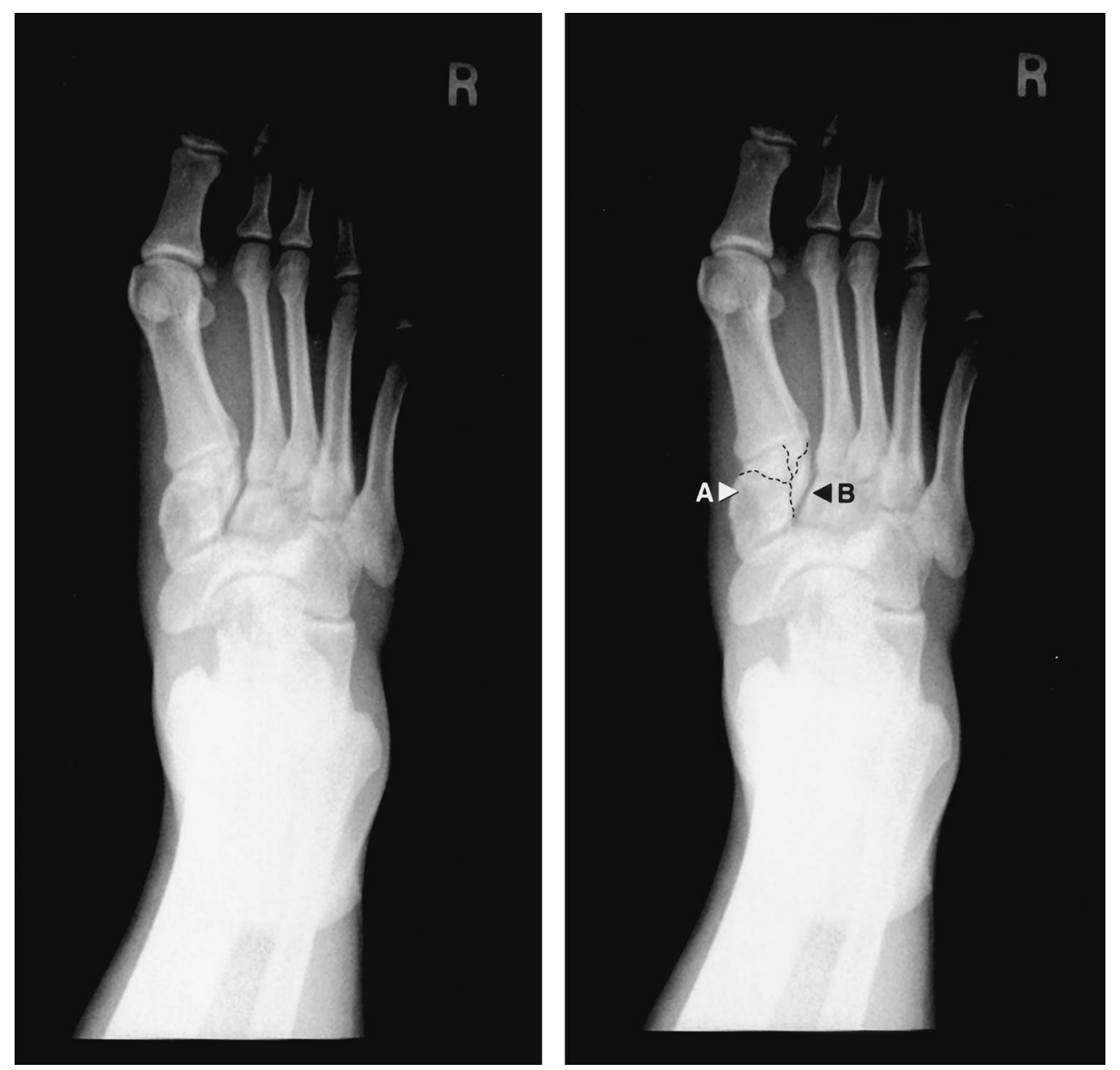

70 JABFP January-February 2003 Vol. 16 No. 1 


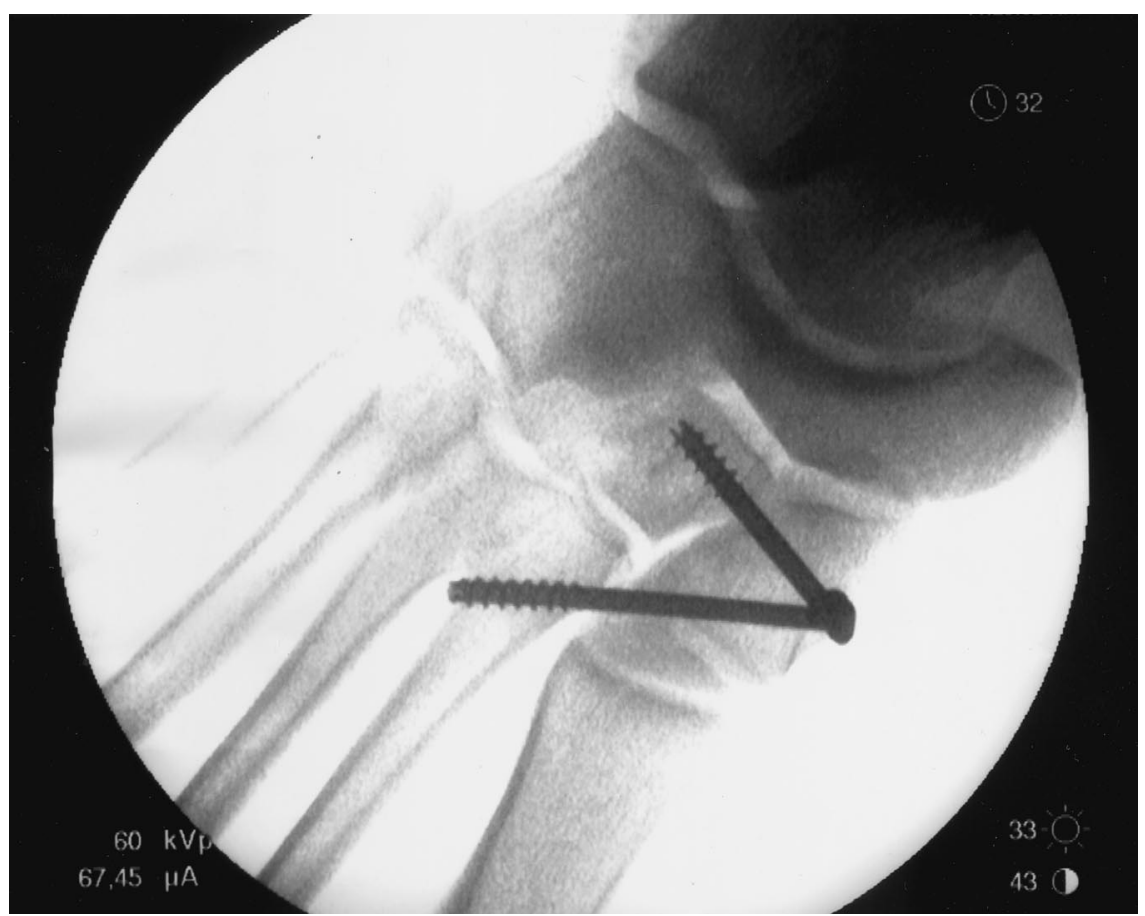

Figure 2. Intraoperative film showing cannulated screw placed from the medial aspect of the medial cuneiform across the Lisfranc articulation into the base of the second metatarsal. A second screw is placed across the medial cuneiform into the middle cuneiform.

on eliciting tenderness over the tarsometatarsal articulations. In addition, stress should be applied to the tarsometatarsal articulations by passive supination and pronation of the forefoot. This test could be the only physical examination maneuver that reproduces discomfort in subtle injuries to this area. It is important to eliminate subtalar motion by maintaining the hindfoot in a position of inversion before performing this test, as a notable amount of supination and pronation of the foot occurs at the subtalar joint.

For patients who complain of foot pain after a classic mechanism of injury and the above findings are observed on physical examination, a strong suspicion of a Lisfranc fracture-dislocation should prompt the clinician to obtain further imaging studies. The physician should also continue to look for this fracture-dislocation when interpreting radiographs, as it has been estimated that $20 \%$ of Lisfranc joint injuries are missed on initial radiography. ${ }^{2,4,12}$ These studies should include weightbearing anteroposterior, lateral, and oblique radiographs of the foot because non-weight-bearing views of the foot can be normal. ${ }^{2,3}$ Foster and Foster ${ }^{13}$ showed that the most consistent radiographic finding in Lisfranc joint injuries was the loss of alignment of the medial border of the second metatarsal and the medial border of the middle cuneiform. If a Lisfranc injury is suspected but not confirmed by radiographs, magnetic resonance imaging or computed tomography of the foot should be considered. ${ }^{5}$

Once the diagnosis is established, the optimal treatment approach and prognosis are subject to controversy. Even though there is little consensus regarding the correlation of long-term outcome to the degree of diastasis between the first two metatarsal heads, current management options are based on the concept that more satisfactory results will ensue from a stable, anatomic reduction of the fracture-dislocation. ${ }^{2,4,12}$ Most investigators have concluded that there is little place for the nonoperative management of Lisfranc fracture-dislocations when a $2-\mathrm{mm}$ or greater diastasis is found between the bases of the first and second metatarsals and medial and middle cuneiforms, because it is difficult to maintain anatomic reduction by closed reduction and immobilization alone. ${ }^{1,2,4,5,7,9,14,15}$

Curtis et $\mathrm{al}^{16}$ recommend surgical reduction for all athletes and active persons. Surgical reduction as soon as possible after the injury is recommended by most orthopaedic surgeons. Trevino and Kodros ${ }^{12}$ 
recommend surgical reduction within the first 24 hours after severe injuries. A delay of 1 to 2 weeks, however, might be appropriate to allow resorption of soft-tissue swelling and does not appear to alter long-term outcomes. ${ }^{5}$ Surgical correction after 6 weeks generally results in poor functional outcomes. $^{12}$

Closed or open reduction with Kirschner wire ( $\mathrm{K}$ wire) internal fixation has historically been used for the treatment of these injuries. ${ }^{14}$ The use of open reduction and internal fixation with $\mathrm{AO}$ screws (meets Arbeitsgemeinschaft für Osteosynthesefragen international standards) has grown increasingly popular for Lisfranc fracture-dislocations. ${ }^{12}$ After screw fixation, most orthopedists recommend immobilization and non-weight-bearing status for 8 to 12 weeks. The screws may be removed at 12 weeks. Full weight bearing is typically not permitted until all hardware is removed.

\section{Conclusion}

It is essential to be highly aware of a possible Lisfranc fracture-dislocation when evaluating patients who have midfoot pain and swelling. Liberal use of weight-bearing radiographs is appropriate when a Lisfranc fracture-dislocation is suspected. Appropriate management, including early surgical consultation, is required to improve the long-term functional outcome.

\section{References}

1. Hesp WL, van der Werken C, Goris RJ. Lisfranc dislocations: fractures and/or dislocations through the tarso-metatarsal joints. Injury 1984;15:261-6.

2. Mantas JP, Burks RT. Lisfranc injuries in the athlete. Clin Sports Med 1994;13:719-30.

3. Burroughs KE, Reimer CD, Fields KB. Lisfranc injuries of the foot: a commonly missed diagnosis. Am Fam Physician 1998;58:118-24.
4. Arntz CT, Hansen ST Jr. Dislocations and fracture dislocations of the tarsometatarsal joints. Orthop Clin North Am 1987;18:105-14.

5. Buzzard BM, Briggs PJ. Surgical management of acute tarsometatarsal fracture dislocation in the adult. Clin Orthop 1998;353:125-33.

6. Hardcastle PH, Reschauer R, Kutscha-Lissberg E, Schoffmann $W$. Injuries to the tarsometatarsal joint. Incidence, classification, and treatment. J Bone Joint Surg Br 1982;64:349-56.

7. Rabin SI. Lisfranc dislocation and associated metatarsophalangeal joint dislocations. A case report and literature review. Am J Orthop 1996;25:305-9.

8. Richter M, Wippermann B, Krettek C, Schratt HE, Hufner T, Therman H. Fractures and fracture dislocations of the midfoot: occurrence, causes and long-term results. Foot Ankle Int 2001;22:392-8.

9. Kuo RS, Tejwani NC, Digiovanni CW, et al. Outcome after open reduction and internal fixation of Lisfranc joint injuries. J Bone Joint Surg Am 2000; 82-A:1609-18.

10. Englanoff G, Anglin D, Hutson HR. Lisfranc fracture-dislocation: a frequently missed diagnosis in the emergency department. Ann Emerg Med 1995;26: 229-33.

11. Perron AD, Brady WJ, Keats TE. Orthopaedic pitfalls in the ED: Lisfranc fracture-dislocation. Am J Emerg Med 2001;19:71-5.

12. Trevino SG, Kodros S. Controversies in tarsometatarsal injuries. Orthop Clin North Am 1995;26: 229-38.

13. Foster SC, Foster RR. Lisfranc's tarsometatarsal fracture-dislocation. Radiology 1976;120:79-83.

14. Myerson MS, Fisher RT, Burgess AR, Kenzora JE. Fracture dislocations of the tarsometatarsal joints: end results correlated with pathology and treatment. Foot Ankle 1986;6:225-42.

15. Myerson M. The diagnosis and treatment of injuries to the Lisfranc joint complex. Orthop Clin North Am 1989;20:655-64.

16. Curtis MJ, Myerson M, Szura B. Tarsometatarsal joint injuries in the athlete. Am J Sports Med 1993; 21:497-502. 